TRANSACTIONS OF THE

AMERICAN MATHEMATICAL SOCIETY

Volume 360, Number 9, September 2008, Pages 4841-4862

S 0002-9947(08)04600-X

Article electronically published on April 24, 2008

\title{
A HILBERT BUNDLE CHARACTERIZATION OF HILBERT C*-MODULES
}

\author{
GEORGE A. ELLIOTT AND KATSUNORI KAWAMURA
}

\begin{abstract}
The category of Hilbert $\mathrm{C}^{*}$-modules over a given $\mathrm{C}^{*}$-algebra is shown to be equivalent to a certain simply described category of Hilbert bundles (i.e., continuous fields of Hilbert spaces) over the space of pure states of the $\mathrm{C}^{*}$-algebra with the zero functional adjoined.
\end{abstract}

1.

In this paper, we shall show that the well-known notion of Hilbert module over a $\mathrm{C}^{*}$-algebra developed by Kaplansky, Rieffel, Paschke, and Kasparov ([9], 16], 14], and [10]; see also [8], 12], and [13]) is equivalent to the notion of Hilbert bundle over the space of pure states of the $\mathrm{C}^{*}$-algebra-provided that the purely topological structure of the Hilbert bundle is augmented with suitable holomorphic and uniform structures. (In the commutative case the additional structure is redundant.) (As in the commutative case, a minor distinction must be made between the cases that the given $\mathrm{C}^{*}$-algebra is unital or not-if not, then the functional zero must be considered at the same time as the pure states, although of course the fibre at zero will always be zero. This is necessary in the non-commutative case in order to express the notion of vanishing at infinity without using compact sets.)

This paper arose out of the simple construction given by the second author in 11] (perhaps also implicit in [14]) of a Hilbert bundle over the pure state space of a $\mathrm{C}^{*}$-algebra corresponding to a given Hilbert module over this $\mathrm{C}^{*}$-algebra - very similar to the construction of Gelfand, Naimark, and Segal of a single Hilbert space for each pure state of the $\mathrm{C}^{*}$-algebra. (The algebra-valued inner product on the Hilbert module yields a scalar-valued one for each pure state on the $\mathrm{C}^{*}$-algebra.)

A question left unresolved in [1] was how to describe the sections of the Hilbert bundle arising from the elements of the Hilbert module. It was pointed out in 11] that these sections respect the natural complex structure on the space of pure states of the $\mathrm{C}^{*}$-algebra (each unitary equivalence class being a Grassmannian), in a natural sense to be described below. This observation will now be complemented by pointing out that the sections arising from the Hilbert module also respect the natural uniform structure on the space of pure states - considered together with the functional zero if the $\mathrm{C}^{*}$-algebra does not have a unit - in the sense that the norm of any section is uniformly continuous. As we shall see, once these compatibilities

Received by the editors August 28, 2006.

2000 Mathematics Subject Classification. Primary 46L05.

The work of the first author was supported by a grant from the Natural Sciences and Engineering Research Council of Canada. 
with the complex and uniform structures of the base space are formalized in terms of natural notions of holomorphic and uniform structures on a Hilbert bundle, the sections arising from the given Hilbert module can be completely characterizedas those bounded sections which are at the same time holomorphic and uniformly continuous. (This is far from immediate, and uses important results from [15] and [3.)

At the same time it is possible to characterize the modules themselves abstractly in terms of this new structure, which it seems reasonable to call that of uniform holomorphic Hilbert bundle, as it incorporates an axiom intertwining the holomorphic and uniform structures. As well as requiring general axioms, this characterization requires the mention of a particular holomorphic Hilbert bundle structure in the case in question, that (each complex manifold component of) the base space is a Grassmannian, namely, either the dual of the classical Hopf bundle (the natural line bundle over a Grassmannian), or a direct sum of copies of this holomorphic Hilbert bundle. (We must require that the restriction of a given uniform holomorphic Hilbert bundle over the space of pure states to each unitary equivalence class - a Grassmannian - or, rather, the restriction of the underlying holomorphic Hilbert bundle - be such a direct sum, in order for the sections to form a Hilbert module over the $\mathrm{C}^{*}$-algebra.) We shall refer to such holomorphic Hilbert bundles as of dual Hopf type.

Theorem. Let $A$ be a $C^{*}$-algebra. Denote by $P_{0}(A)$ the set of extreme points of the convex set of positive linear functionals on $A$ of norm at most one (in other words, the set $P_{0}(A)$ of pure states of $A$ together with the functional 0$)$. Consider $P_{0}(A)$ with its natural uniform structure (determined by the seminorms arising from evaluation at the elements of $A$ ) and with its natural complex structure (determined by realizing each unitary equivalence class of pure states as the projective space based on the Hilbert space arising from one of them-of course the complex structure is independent of the choice of representative pure state).

The category of right Hilbert A-modules is equivalent to the category (described in Sections 2 to 4 below) of uniform holomorphic Hilbert bundles over $P_{0}(A)$ of dual Hopf type.

More specifically, let $X$ be a right Hilbert A-module (definition recalled below). For each $f \in P_{0}(A)$ denote by $H(X)_{f}$ the Hilbert space arising from the positive sesquilinear form on $X$ obtained by composing the A-valued inner product with $f$. The sections of the bundle $\left(H(X)_{f}\right)_{f \in P_{0}(A)}$ corresponding to the elements of $X$ constitute the bounded uniformly continuous holomorphic sections of a unique uniform holomorphic Hilbert bundle, $H(X)$, over $P_{0}(A)$, necessarily of dual Hopf type. (The axioms for this mathematical structure are given in Section 4 below.)

Let $H$ be a uniform holomorphic Hilbert bundle over $P_{0}(A)$, of dual Hopf type (see Section 4 below). The vector space of bounded uniformly continuous holomorphic sections of $H$ admits a unique structure of right Hilbert $A$-module, $X(H)$, such that the uniform holomorphic Hilbert bundle $H(X(H)$ ) is equal to $H$ (in the natural sense).

If a right Hilbert $A$-module $X$ is given, then, with $X$ viewed as sections of $H(X)$ as in the construction of this Hilbert bundle, $X(H(X))$ is equal to $X$ not only as a set (which it is by definition) but also as a Hilbert $A$-module.

The correspondences $X \mapsto H(X)$ and $H \mapsto X(H)$ described above are functorial, with respect to bounded $A$-module maps between Hilbert $A$-modules and morphisms 
between Hilbert bundles (i.e., fibre-preserving maps between the total spaces, linear and bounded in each fibre and with an overall bound on the norm) respecting bounded uniformly continuous holomorphic sections.

These functors give rise to an equivalence of categories. In fact, this is true in a strong sense: the composed functors $X \mapsto X(H(X))$ and $H \mapsto H(X(H))$ may already be viewed as the identity functors (no natural transformations of functors needed).

\section{UNiform HilBert BUNDLES}

Recall from [6] and [9] (see also [7, [5], [18, and [4]) that a family $H=\left(H_{t}\right)_{t \in T}$ of Hilbert spaces indexed by a topological space $T$ is said to be a Hilbert bundle (or continuous field of Hilbert spaces) over $T$ if there is specified a vector space of sections, mapping onto each fibre Hilbert space $H_{t}$-in other words, exhausting fibres - which consists of sections with continuous norm (a section $\xi$ is said to have continuous norm if the function $T \ni t \mapsto\|\xi(t)\| \in \mathbb{R}$ is continuous) and is maximal with this property. In this case the specified sections are referred to as the continuous sections (of the Hilbert bundle $H$ ).

Analogously, let us introduce the notion of uniform Hilbert bundle (or uniformly continuous field of Hilbert spaces), as follows. Let $H=\left(H_{u}\right)_{u \in U}$ be a family of Hilbert spaces indexed by a uniform space $U$ (for instance, $U$ might be the uniform space underlying a metric space). Let us say that $H$ has the structure of uniform Hilbert bundle over $U$ if there is specified a vector space of sections, exhausting fibres, which consists of sections with uniformly continuous norm (a section $\xi$ will be said to have uniformly continuous norm if the function $U \ni u \mapsto\|\xi(u)\| \in \mathbb{R}$ is uniformly continuous) and is maximal with this property. In this case let us refer to the specified sections as the uniformly continuous sections (of the uniform Hilbert bundle $H$ ).

Note that a structure of uniform Hilbert bundle over $U$ determines a unique topological Hilbert bundle over the topological space underlying $U$ (such that the uniformly continuous sections are among the continuous sections). If $U$ is compact as a topological space, so that the uniform structure on $U$ (giving rise to this topology) is unique and any continuous function from $U$ to another uniform space is uniformly continuous, then the Hilbert bundle structure determined by a uniform Hilbert structure over $U$ coincides with it, in the sense that every continuous section is uniformly continuous. In other words, the notion of uniform Hilbert bundle only differs from that of (topological) Hilbert bundle when the base space is not compact.

Note that, while, as is well known, a given linear space of sections with continuous norm, exhausting fibres, with respect to a family of Hilbert spaces $\left(H_{t}\right)_{t \in T}$ indexed by a topological space $T$, determines a unique structure of Hilbert bundle - i.e., is contained in a unique maximal linear space of sections with continuous norm (the space of all sections the sum of which with any one of the given sections has continuous norm) - , if $T$ is replaced by a uniform space $U$, and a linear space of sections with uniformly continuous norm, exhausting fibres, is given, it is not clear whether this is contained in a unique maximal such subspace. (This is equivalent to the question whether, for any two sections, the sum of each of which with an arbitrary one of the given sections has uniformly continuous norm, the sum of these sections also has this property - equivalently, always itself has uniformly continuous norm.) 
Naturally, instead of Hilbert spaces as fibres one may consider Banach spaces (and speak of Banach bundles and uniform Banach bundles). (We shall not do this here.)

Other questions also arise concerning uniform Hilbert bundles. Recall, for instance, that to specify a (topological) Hilbert bundle it is enough to specify a linear space of sections with continuous norm the image of which in each fibre is dense. The space of all sections the sum of which with each of the given sections has continuous norm will then map onto each fibre - i.e., will exhaust fibres - and will in fact be the space of all continuous sections with respect to a structure of Hilbert bundle. (To prove that the larger space, say $\Gamma$, of sections maps onto each fibre, write an arbitrary given vector in a given fibre as the sum of a sequence of vectors in the dense subspace the norms of which are summable (for instance, at most $2^{-n}$ from some point on). Note that each of these vectors is the image not only just of some section in $\Gamma$ but of some section in $\Gamma$ the norm of which at each point is at most the norm of the image in the fibre in question. To see this, it is enough to note that $\Gamma$ is closed under pointwise multiplication by continuous scalar-valued functions (since continuity of the norm is a local property). Given a continuous section, $\xi$, not equal to zero in a given fibre, say at $t_{0} \in T$, the product of $\xi$ and the inverse of the continuous non-vanishing function

$$
t \mapsto\left\|\xi\left(t_{0}\right)\right\|^{-1} \max \left(\left\|\xi\left(t_{0}\right)\right\|,\|\xi(t)\|\right)
$$

is equal to $\xi$ at $t_{0}$ and has norm at any $t \in T$ at most its norm at $t_{0}$. The sum of this sequence of sections then exists in the supremum norm, and yields a section in $\Gamma$ and mapping onto the given vector in the given fibre.) (It remains to note that $\Gamma$ is maximal as a space of sections with continuous norm, but this is immediate.)

It is not clear if to specify a uniform Hilbert bundle it is enough to specify a linear space of sections with uniformly continuous norm, mapping onto a dense subset of each fibre even if one specifies maximality. (In the presence of maximality, it would be enough to know that, in analogy with the continuous case, one could multiply pointwise by a uniformly continuous scalar-valued function-the given space of sections would then, as in the continuous case, map onto each fibre.)

\section{Holomorphic Hilbert Bundles}

Let $T$ be a (not necessarily connected) complex manifold, based on a complex Hilbert space - or, rather, on an open subset of a complex Hilbert space - at each point, and let $H=\left(H_{t}\right)_{t \in T}$ be a family of complex Hilbert spaces. Suppose that for every $t \in T$ there is a neighbourhood of $t$ such that $H_{s}$ is isomorphic to $H_{t}$ for all $s$ in this neighbourhood. (Equivalently - although no such reformulation is possible for Banach spaces - the Hilbert space dimension of $H_{t}$ is continuous, in other words, locally constant.) Let us say that $H$ has a structure of (locally trivial) holomorphic Hilbert bundle if the set-theoretic union (the total space) $\bigcup_{t \in T} H_{t}$ is endowed with a structure of complex manifold which, over an open neighbourhood of each $t \in T$, is isomorphic to the Cartesian product complex structure on the Cartesian product of $H_{t}$ with this neighbourhood of $t$ in $T$, by means of a set-theoretic bijection which preserves fibres and is linear and bounded on each fibre, with the norm of the linear operator varying continuously over the neighbourhood - and if, furthermore, the bounded holomorphic sections exhaust fibres. (We shall only consider locally trivial holomorphic Hilbert bundles - although it might be interesting to consider 
a more general notion of holomorphic Hilbert bundle, including, for instance, subbundles and arbitrary direct sums - cf. below - of locally trivial bundles.)

Note that, implicit in the concept of complex manifold, and also that of (locally trivial) holomorphic Hilbert bundle just introduced (in analogy with the well-known case of finite-dimensional vector spaces, not assumed to be endowed with a fixed Hilbert space structure, as fibres) is the notion of holomorphic function from an open subset of one Hilbert space to another Hilbert space. As is well known (see [1]), there are several equivalent formulations of this notion. Let us use in the first instance (we shall use the others implicitly) the following definition: the function should be continuous, and Gâteaux differentiable (i.e., the restriction to each onedimensional complex affine subspace should be holomorphic (= differentiable) as a function of one complex variable). (While some proof as needed with this criterion that a composition of holomorphic functions is holomorphic, it is of course true.)

It is important to note that, as a consequence of the axiom that the bounded holomorphic sections of a holomorphic Hilbert bundle exhaust fibres, the bounded holomorphic sections in fact determine the holomorphic Hilbert bundle. In anticipation of Section 4, let us in fact prove the following stronger statement:

any linear subspace of bounded holomorphic sections exhausting fibres determines the holomorphic Hilbert bundle structure, in the natural sense.

More explicitly, let us show that any set-theoretic bijection between the total spaces of two (locally trivial) holomorphic Hilbert bundles over the base space $T$, preserving fibres and reducing to a Hilbert space isomorphism (isometric) between corresponding fibres - or even just a bicontinuous linear mapping, with the norms of the mapping and its inverse bounded over all fibres - or even just locally boundedand taking a specified linear subspace of bounded holomorphic sections of the first bundle exhausting fibres onto a similar subspace of sections of the second bundle, is an isomorphism - not necessarily isometric - of holomorphic Hilbert bundles, i.e., is holomorphic (together with its inverse - but this is automatic), as a map between the two total spaces considered as complex manifolds according to the definition of a (locally trivial) holomorphic Hilbert bundle. In particular, this includes the case of the identity map on the set-theoretic total space of a given holomorphic Hilbert bundle, with a specified linear space of sections as above, which are also holomorphic with respect to a second holomorphic Hilbert bundle structure.

To show this, it is enough (since we are only considering - or even defininglocally trivial holomorphic Hilbert bundles) to show that the holomorphic Hilbert bundle structure is determined in the trivial case. What this amounts to is the following statement: If $T$ is a complex manifold based at each point on an open subset of a complex Hilbert space, and if $K$ is a fixed complex Hilbert space, and if the Cartesian product $T \times K$ is bijectively mapped onto itself in a way which takes each fibre onto itself, by means of a bounded linear mapping, with norm bounded over all fibres, and if this mapping takes a fixed linear space of bounded holomorphic sections of the trivial holomorphic Hilbert bundle $T \times K$, exhausting each fibre, onto itself, then this map must be holomorphic with respect to the Cartesian product complex structure on $T \times K$.

Let $F$ be such a map (as just described). Let us show that $F$ is continuous, and that - assuming, as we may, that $T$ is in fact an open subset of a Hilbert space - the restriction of $F$ to each complex line is holomorphic (in the usual sense). 
First, to see that $F$ is continuous, it is enough to note that $F$ preserves fibres and is linear and bounded on each fibre (with the norm of the linear transformation bounded at least in a neighbourhood of each point of $T$ ), and, furthermore, takes a collection of continuous sections exhausting fibres into continuous sections. One deduces first that $F$ takes arbitrary continuous sections into continuous sections. (Indeed, if $f$ is an arbitrary continuous section-i.e., continuous function from $T$ to $K$-and if $t_{0} \in T$, to prove that $F \circ f$ is continuous at $t_{0}$, suppose first that $f\left(t_{0}\right)=0$. Then $f(t)$ tends to 0 as $t$ tends to $t_{0}$, and since the operator $F(t)$ defined by $F$ in the fibre at $t$ is uniformly bounded over all fibres it follows that $F(t) f(t)=(F \circ f)(t)$ also tends to 0 as $t$ tends to $t_{0}$. If now $f\left(t_{0}\right) \neq 0$, choose according to the hypothesis a continuous section $g$ such that $g\left(t_{0}\right)=f\left(t_{0}\right)$ and $F \circ g$ is continuous. Since $(f-g)\left(t_{0}\right)=0$ it follows by the special case just considered that $F \circ(f-g)$ is continuous at $t_{0}$. Hence $F \circ f(=F \circ(f-g)+F \circ g)$ is continuous at $t_{0}$.) It follows that $F$ is continuous. (If $\left(t_{n}, \xi_{n}\right)$ converges to $(t, \xi)$ in $X \times K$, to prove that $F\left(t_{n}, \xi_{n}\right)$ converges to $F(t, \xi)$, since $\left\|F\left(t_{n}\right)\right\|$ is bounded it is enough to suppose that $\xi_{n}=\xi$ for all $n$. Then the points $\left(t_{n}, \xi_{n}\right)$ lie on a continuous section (a constant one), and so also the points $F\left(t_{n}, \xi_{n}\right)$ do, as desired.)

Now let $L$ be a complex line in $T \times K$ and let us show that the restriction of $F$ to $L$ is holomorphic in the usual sense - namely, differentiable with respect to the complex variable. (Note that we are not using in any fundamental way that lines are special subsets, as we are just using one criterion for the map $F$ to be holomorphic.)

The case that $F$ is contained in a single fibre is trivial, as $F$ is complex linear on that fibre. In the other case, let $(t, \xi)$ be a point in $L$ and let us show that the restriction of $F$ to $L$ is differentiable at $(t, \xi)$. Choose a holomorphic section $f$ such that $F \circ f$ is holomorphic and such that $f(t)=\xi$. (Such a section exists by hypothesis.) Changing notation, we may suppose that $t=0$. Denote by $L_{1}$ the coordinate projection of the line $L$ into $T$, and, choosing a non-zero vector in $L_{1}$ identify $L_{1}$ with the complex plane. In this way $L$ becomes the function $z \mapsto \xi+z \lambda$ for some $\lambda \in K$. Considering the restriction of $f$ to $L_{1}$ as a function on the complex plane (in particular, as a function rather than a section, i.e., with values in $K$ rather than $T \times K)$ we then have

$$
f(z)=f(0)+z f^{\prime}(0)+\mathrm{o}(|z|),
$$

where $f(0)=\xi$ and $f^{\prime}(0) \in K$. Choose a holomorphic section $g$ such that $F \circ g$ is holomorphic and $g(0)=\lambda-f^{\prime}(0)$. (Again, such a section exists by hypothesis.) Restricting $g$ to $L_{1}$, and considering it as a function with values in $K$, we have

$$
g(z)=g(0)+z g^{\prime}(0)+\mathrm{o}(|z|)
$$

whence

$$
z g(z)=z g(0)+\mathrm{o}(|z|)
$$

We must show that the function on the complex plane

$$
z \mapsto F(z, \xi+z \lambda)
$$

is differentiable (as a function of a complex variable). Since the functions $z \mapsto$ $F(z, f(z))$ and $z \mapsto F(z, g(z))$ are differentiable, it follows that the function

$$
z \mapsto F(z, f(z)+z g(z))=F(z, f(z))+z F(z, g(z))
$$


is differentiable. Since

$$
\begin{aligned}
f(z)+z g(z) & =f(0)+z f^{\prime}(0)+z g(0)+\mathrm{o}(|z|) \\
& =\xi+z \lambda+\mathrm{o}(|z|)
\end{aligned}
$$

and since we may suppose that $F$ is a contraction in each fibre,

$$
\begin{aligned}
F(z, f(z)+z g(z)) & =F(z, \xi+z \lambda+\mathrm{o}(|z|)) \\
& =F(z, \xi+z \lambda)+\mathrm{o}(|z|) .
\end{aligned}
$$

In other words, $z \mapsto F(z, \xi+z \lambda)$ is within $\mathrm{o}(|z|)$ of a differentiable function, and so is differentiable $($ at $(0, \xi)=(t, \xi))$ itself, as desired.

The main example of a holomorphic Hilbert bundle that we shall need to consider is what is well known at least in the finite-dimensional case as the dual Hopf bundle - the dual of the natural line bundle over the projective space corresponding to a given Hilbert space. As is well known (and will be shown for the convenience of the reader below) the bounded holomorphic sections of this bundle arise in a natural way from the vectors in the Hilbert space. An equivalent definition is to look at the dual space of the given Hilbert space instead, in other words, at $B(K, \mathbb{C})$ where $K$ denotes the Hilbert space, and consider the base space as the Grassmannian instead of the projective space, in which case the fibres are the family of one-dimensional Hilbert spaces

$$
(B(K, \mathbb{C}) e ; e \text { a rank-one projection on } K),
$$

and the bounded holomorphic sections the maps

$$
e \mapsto t e \in B(K, \mathbb{C}) e, \quad t \in B(K, \mathbb{C}) .
$$

Let us check that the projective space associated to a Hilbert space $K$, i.e., the space of one-dimensional subspaces of $K$, does have a natural complex structure, with the coordinate maps arising from continuous linear functionals, and that the sections described above (arising from the elements of $K$ ) are precisely the bounded holomorphic sections of a (locally trivial) holomorphic Hilbert bundle structure on the canonical set-theoretic line bundle over this space. (By the general uniqueness result established above, such a holomorphic Hilbert bundle structure is unique, in the natural sense.)

First, the complex structure; we must verify that the coordinate maps arising from non-zero continuous linear functionals - on the space of all one-dimensional subspaces - each non-zero functional considered to define a coordinate map on the subset of one-dimensional subspaces not contained in its kernel, by the specification of the hyperplane of vectors on which this functional is equal to one (a crosssection of the set of one-dimensional subspaces not killed by the functional) - are compatible. In other words, given two continuous linear functionals $f_{1}$ and $f_{2}$ on $K$, we must show that, on the open subset of the hyperplane $R_{1}=\left\{\kappa \in K ; f_{1}(\kappa)=1\right\}$ where the map from this hyperplane to one-dimensional subspaces and then back to the hyperplane $R_{2}=\left\{\kappa \in K ; f_{2}(\kappa)=1\right\}$ is defined, i.e., on the subset of $R_{1}$ on which $f_{2}$ is non-zero, this map is holomorphic. But this map is just the map

$$
R_{1} \backslash \operatorname{ker} f_{2} \ni \kappa \mapsto f_{2}(\kappa)^{-1} \kappa \in R_{2} \backslash \operatorname{ker} f_{1}
$$

which is holomorphic since $f_{2}$ is.

The verification of our axioms for a holomorphic Hilbert bundle, for the dual Hopf bundle associated to the Hilbert space $K$, is similar. First of all, each of 
the hyperplanes considered above defines a trivialization of the Hopf (or tautological) bundle over the set of one-dimensional subspaces intersecting it, by means of bounded linear mappings with continuously varying norm, which gives it a complex structure over that open subset of the base space - and equally for the dual Hopf bundle-, and the discrepancy maps for two such hyperplanes are holomorphic, so the complex structure exists globally. While the Hopf bundle has no global holomorphic sections (see [2, pages 6 and 7), for every vector in $K$ (or, rather, for every bounded linear functional on $K$ ) one obtains a global bounded holomorphic section of the dual bundle, and these clearly exhaust the (one-dimensional) fibres.

The bounded holomorphic sections of the dual Hopf bundle associated to $K$ consist exactly of the families of functionals on one-dimensional subspaces corresponding to fixed continuous functionals on all of $K$ (even if $K$ is a Banach space). This is shown in 2] (page 7) in the case that $K$ is finite-dimensional; let us check this in the present case, that $K$ is arbitrary (even a Banach space). Given a holomorphic section, consider the function $f$ on $K$ which to any $\kappa \in K$ associates the value on $\kappa$ of the functional given by the section at the one-dimensional subspace generated by $\kappa$, if $\kappa \neq 0$, and 0 if $\kappa=0$. Clearly $f(\lambda \kappa)=\lambda f(\kappa)$. Let us show that $f$ is holomorphic. The restriction of $f$ to a complex line in $K$ is linear if the line is a subspace, and if the line does not go through the origin, embedding it in a hyperplane also not containing the origin, which by definition trivializes the dual Hopf bundle over it, we have that $f$ is holomorphic because the given section is assumed to be holomorphic. We must check that $f$ is continuous, but this is true on $K \backslash 0$ by the assumption that the given section is continuous - and that each hyperplane not containing 0 covers an open subset of the projective space-, and is true at 0 by homogeneity and the assumption that the given section is boundedwhich implies that $f$ is bounded on the unit ball of $K$. Finally, from the facts that $f$ is holomorphic and homogeneous, it follows immediately that $f$ is linear (it is enough to consider the case $K=\mathbb{C}^{2}$; consider the power series expansion of $f$ ). (The assumption of boundedness of the given section is in fact redundant, even if $K$ is infinite-dimensional, because in any case it must have continuous norm, since by definition a holomorphic section of a holomorphic Hilbert bundle is a continuous section, and if $K$ is finite-dimensional the projective space is compact and so the norm of the section (as a function on the space of fibres) is bounded. Hence by the proof in the bounded case, the restriction of the section to the space of one-dimensional subspaces contained in each fixed finite-dimensional subspace of $K$ arises from a linear functional - and so (as the various functionals must be compatible) one obtains a possibly unbounded linear functional on all of $K$, giving rise to the given holomorphic section. However, as shown above, even if the given holomorphic section is not assumed to be bounded, the scalar-valued function on $K \backslash 0$ associated to it as above must still be continuous; if the section arises from a linear functional, then this scalar-valued function is just the functional itself-and if a linear functional on $K$ is continuous on $K \backslash 0$ it must be bounded as a functional.)

We shall also need to consider direct sums of copies of the dual Hopf bundleusually of infinitely many copies! Let us say that a holomorphic Hilbert bundle obtained in this way is of dual Hopf type. The direct sum is defined in a natural way, but it should be pointed out that it is not clear how to define the direct sum of an arbitrary family of holomorphic Hilbert bundles, although the direct sum of the underlying (topological) Hilbert bundles is easy to define (just consider the 
pointwise direct sum of fibres, and note that the finite sums of continuous sections in the various summands form a linear space of sections of continuous norm which maps onto a dense subspace of each fibre, and which therefore as pointed out in Section 2 (see also [4]) is contained in a unique maximal linear space of sections of continuous norm).

The difficulty in defining the direct sum of holomorphic Hilbert bundles in general is that, first, it may not be possible to assure a locally trivial holomorphic structure in an infinite direct sum if the intersection of neighbourhoods over which the components are trivial is just a single point - this is overcome by assuming only one isomorphism type of component-and, second, even if the holomorphic sections exhaust fibres in each component, it is not clear that they do for the direct sum - unless the fibres are all one-dimensional. In the case of a sum of holomorphic line bundles, given an arbitrary vector in the direct sum of the fibres at one point, its Fourier expansion in terms of orthogonal copies of a fixed unit vector in the model component can be extended to a convergent sum of orthogonal copies of a fixed holomorphic section of the given one-dimensional holomorphic Hilbert bundle. This sum is pointwise convergent (in each direct sum fibre), and, in fact, is uniformly convergent if we assume, as we may according to our definition of holomorphic Hilbert bundle, that the chosen section of the line bundle is bounded. We then easily confirm the holomorphic property of the limit section, and note that it is bounded and maps onto the given vector in the specified fibre, as desired.

As mentioned earlier, it is an interesting question whether the notion of holomorphic Hilbert bundle can be defined beyond the locally trivial case, in such a way that, for instance, arbitrary direct sums exist.

Holomorphic Hilbert bundles of dual Hopf type may also be described as follows: As before, the base space should be the Grassmannian on a Hilbert space, say $H_{1}$. Given a Hilbert space $H_{2}$, consider the family of Hilbert spaces

$$
H=\left(B\left(H_{1}, H_{2}\right) e ; e \text { is a rank-one projection on } H_{1}\right) .
$$

Consider the family of sections

$$
e \mapsto t e, t \in B\left(H_{1}, H_{2}\right) .
$$

This is the space of bounded holomorphic sections of a (unique) holomorphic Hilbert bundle, which is isomorphic to the direct sum of a number of copies of the dual Hopf bundle (over the Grassmannian on $H_{1}$ ) equal to the dimension of $H_{2}$.

\section{Uniform holomorphic Hilbert Bundles}

Let $T$ be a complex manifold, based on a complex Hilbert space or on an open subset of a complex Hilbert space at each point. Let $H=\left(H_{t}\right)_{t \in T}$ be a (locally trivial) holomorphic Hilbert bundle over T. (See Section 3.)

Let there be given a uniform structure on $T$-not necessarily related in any simple way to the complex structure. (In our examples, arising from Hilbert $\mathrm{C}^{*}$-modules, the topology underlying the uniform structure is comparable withcoarser than - that underlying the complex structure, coinciding with it in the case that the $\mathrm{C}^{*}$-algebra is finite-dimensional.) (It would be interesting to determine exactly when these two topologies, in the case arising from a $\mathrm{C}^{*}$-algebra, coincide.)

Let us say that $H$ has a structure of uniform holomorphic Hilbert bundle if there is given a linear subspace of the bounded holomorphic sections of $H$ with uniformly 
continuous norm, maximal with this property, and exhausting each fibre. Let us call this space of sections the bounded uniformly continuous holomorphic sections.

In this case, there exists by Zorn's Lemma a structure of uniform Hilbert bundle with fibres $\left(H_{t}\right)_{t \in T}$ such that the given subspace is precisely the space of bounded sections which are both holomorphic with respect to the holomorphic Hilbert bundle structure and uniformly continuous with respect to the uniform Hilbert bundle structure. We do not know if this uniform Hilbert bundle structure is unique. (Note that there does exist a unique structure just of Hilbert bundle on $H$ such that the bounded uniformly continuous holomorphic sections are continuous - as these exhaust fibres and have continuous norm - see Section 2.)

More to the point is that a uniform holomorphic Hilbert bundle does determine the holomorphic Hilbert bundle entering into its definition. (By Section 3, a (locally trivial) holomorphic Hilbert bundle structure is determined uniquely - in the strongest possible sense - by (virtually) any family of holomorphic sections which exhausts fibres.) (Actually, in our examples, arising from Hilbert $\mathrm{C}^{*}$-modules, the holomorphic Hilbert bundle structure is determined in an even stronger way by the bounded uniformly continuous holomorphic sections - namely, by Kadison transitivity, any bounded holomorphic section coincides with a bounded uniformly continuous holomorphic section on the sub-Grassmannian corresponding to any finite-dimensional subspace of the Hilbert space - also at the same time on finitely many of the Grassmannian components of the base space.)

Note that, even though the holomorphic Hilbert bundle underlying a given uniform holomorphic Hilbert bundle (i.e., the unique holomorphic Hilbert bundle with the given fibres and with respect to which the given uniformly continuous holomorphic sections are holomorphic - cf. above) is locally trivial, as is also the underlying (topological) Hilbert bundle, it would not even appear to have a meaning for the uniform holomorphic Hilbert bundle to be locally trivial (as uniform continuity is not a local phenomenon). (At any rate, for a uniform Hilbert bundle - in contrast to the case of a topological or holomorphic Hilbert bundle - a local trivialization, if it existed, would not necessarily determine the global (uniformly continuous) sections.)

Let us say that a uniform holomorphic Hilbert bundle is of dual Hopf type if the (uniquely determined) underlying holomorphic Hilbert bundle is of dual Hopf type (see Section 3-this of course only makes sense if the base space is a disjoint union of complex projective spaces). (As in Section 3, even for the examples of dual Hopf type, where we should perhaps consider only the second description because it is not clear what abstract direct sum to take, the notion of uniform holomorphic Hilbert bundle has a Banach space analogue.)

\section{Proof of Theorem 1}

Let $X$ be a right Hilbert $A$-module, i.e. (see [14]), a right $A$-module with an $A$-valued biadditive functional

$$
X \times X \ni(x, y) \mapsto(x \mid y)=(x \mid y)_{A} \in A
$$

such that

$$
\begin{aligned}
(x \mid y a) & =(x \mid y) a, x, y \in X, a \in A \\
(y \mid x) & =(x \mid y)^{*}, x, y \in X
\end{aligned}
$$


and

$$
(x \mid x) \geq 0, x \in X
$$

with $(x \mid x)=0$ only if $x=0$, and such that $X$ is complete in the norm $x \mapsto\|x\|:=$ $\|(x \mid x)\|^{1 / 2}$ (see [14]). (The proof that the functional $x \mapsto\|(x \mid x)\|^{1 / 2}$ is a norm consists of recognizing it as the supremum of the functionals $x \mapsto(f((x \mid x)))^{1 / 2}$ with $f$ a state of $A$, each one of which is a seminorm as $(x, y) \mapsto f((x \mid y))$ is a semi-inner-product-i.e., an inner product modulo null vectors.)

Consider the family - or bundle, in the set theory sense - of Hilbert spaces $H(x)$ $:=\left(H(X)_{f}\right)_{f \in P_{0}(A)}$, with $H(X)_{f}$ as defined above - the Hilbert space completion of the pre-Hilbert space associated to the positive sesquilinear form $(x, y) \mapsto f((x \mid y))$ on $X$ (formed by dividing out by the subspace of null vectors). In fact, by the Kadison transitivity theorem, as is already well known in the case of the module $A$ itself, this pre-Hilbert space is already complete - and we shall use this (see Section 4). Let us show that the sections of $H(X)$ corresponding to the elements of $X$ (each $x \in X$ having a natural image in $H(X)_{f}$ for each $f \in P_{0}(A)$ ) are precisely the bounded uniformly continuous holomorphic sections of a uniform holomorphic Hilbert bundle over $P_{0}(A)$ with fibres $H(X)_{f}, f \in P_{0}(A)$ (where $P_{0}(A)$ is considered as mentioned with its natural complex structure and uniform structure - note that these two structures give rise to different topologies in general). Let us show also that this uniform holomorphic Hilbert bundle structure - which is unique by Section 4 (determined by definition by its bounded uniformly continuous holomorphic sections) is of dual Hopf type.

Perhaps surprisingly, in order to do this, it would appear to be necessary to proceed rather indirectly - first constructing just by Zorn's Lemma some uniform holomorphic Hilbert bundle, with fibres $H(X)_{f}, f \in P_{0}(A)$, having the space of sections in question as a subspace of its space of uniformly continuous holomorphic sections, and then using the converse statement of the theorem-which, although stated later, must therefore be proved first - to endow the space of all bounded uniformly continuous holomorphic sections of this bundle with the structure of Hilbert $A$-module (using the work of Brown referred to above), in such a way that - as we must show! - the subspace of sections in question, with its given $A$ module and $A$-valued inner product space structures, is a substructure. Then (on account of the work of Pedersen referred to above) it will be possible to deduce that this submodule is equal to the whole module - in other words, that the space of sections in question (the given module) is equal to the space of all bounded uniformly continuous holomorphic sections of the constructed uniform holomorphic Hilbert bundle - which, it should be pointed out (see Section 4), is determined uniquely by this data (the family of fibres $\left(H(X)_{f}\right)_{f \in P_{0}(A)}$ together with the space of all bounded uniformly continuous holomorphic sections). The fact (to be proved) that this bundle is of dual Hopf type will have already been proved at the time of its construction, in order to make possible the application of the converse statement of the theorem.

The first step in the proof, then, must be to start with a uniform holomorphic Hilbert bundle $H=\left(H_{f}\right)_{f \in P_{0}(A)}$, of dual Hopf type, and show that the space $X(H)$ of bounded uniformly continuous holomorphic sections of $H$ admits, in a natural way, a structure of right Hilbert $A$-module. (To establish the full conclusion of the theorem in this connection, we must show eventually that with this structure of 
Hilbert $A$-module on $X(H)$, the corresponding uniform holomorphic Hilbert bundle $H(X(H)$ ) - which has not yet even been constructed! (cf. above) - is equal to $H$ (but this will follow from general properties of this class of bundles - in fact, from the definition - once we know that the fibres and the spaces of bounded uniformly continuous holomorphic sections are the same). We must also show that this property determines the Hilbert $\mathrm{C}^{*}$-module structure on $X(H)$ uniquely.)

Recall from Section 4 that the condition that $H$ be of dual Hopf type is just a condition on the holomorphic structure of $H$, i.e., on the structure of $H$ as a holomorphic Hilbert bundle over $P_{0}(A)$, this space endowed simply with its natural complex structure, as the disjoint union of Grassmannians (projective spaces, based on complex Hilbert spaces). Recall from Section 3 that a holomorphic Hilbert bundle over the Grassmannian of the Hilbert space $K$ is of dual Hopf type precisely when for some Hilbert space $K^{\prime}$ it is (isomorphic to) the holomorphic Hilbert bundle with space of sections $B\left(K, K^{\prime}\right)$ and fibres $B\left(K, K^{\prime}\right) e$, e a rank one projection in $B(K)$. (Thus, the element $s$ of $B\left(K, K^{\prime}\right)$ is understood to give rise to the section $e \mapsto s e$.

Recall, again from Section 4, that a structure of uniform holomorphic Hilbert bundle, based on an underlying structure of holomorphic Hilbert bundle (see Section 3), consists of a linear subspace of the bounded holomorphic sections - to be referred to as the bounded uniformly continuous holomorphic sections - which is maximal with the property that all its elements have uniformly continuous norm (the norm in a fibre considered as a function on the base space, that is), with respect to a specified uniform structure on the base space. There is also an additional hypothesis - to ensure that the bounded uniformly continuous holomorphic sections provide complete information concerning the uniform holomorphic Hilbert bundle structure - namely, that these sections exhaust fibres.

Consider $A$ as a right Hilbert module over itself, i.e., with the $A$-valued inner product $(a, b) \mapsto a^{*} b$. For each $f \in P_{0}(A)$, consider the Hilbert space $H(A)_{f}$ referred to in the statement of the theorem; it is of course the Gelfand-NaimarkSegal Hilbert space associated to the positive functional $f$. (Note that we are considering all inner products as linear in the second variable.) In particular, as is well known, $A$ acts naturally (on the left) on the Hilbert space $H(A)_{f}$ and this representation is irreducible (if $f$ is non-zero).

For each fixed unitary equivalence class in $P_{0}(A)$, pick a representative element, and consider the corresponding Hilbert space and representation of $A$ as above. Note that for every pure state $g$ of $A$ in this equivalence class there exists a unique projection of rank one $e_{g}$ on this Hilbert space such that

$$
e_{g} a e_{g}=g(a) e_{g}, a \in A,
$$

and that every rank one projection arises in this way.

The set of projections of rank one on this Hilbert space, or, alternatively, the space of one-dimensional subspaces, with its natural (quotient) complex structure, is in fact the Grassmannian naturally associated to this unitary equivalence class, which was referred to above. (Note that the identification of the equivalence class with the Grassmannian is independent of the choice of representative in the equivalence class, as two projections of rank one corresponding to the same pure state in two different-but unitarily equivalent - irreducible representations must correspond by any Hilbert space isomorphism intertwining the two representations.) 
(In particular, the complex structure on $P_{0}(A)$ does not depend on the choice of representatives of unitary equivalence classes.)

For each fixed unitary equivalence class in $P_{0}(A)$, now realized as the Grassmannian on a Hilbert space, on which $A$ acts (on the left), choose a second Hilbert space such that the given holomorphic Hilbert bundle of dual Hopf type underlying $H$, restricted to this equivalence class, is isomorphic to that associated to the space of bounded linear operators from the first Hilbert space to the second, with (cf. above) holomorphic sections

$$
e \mapsto t e, t \text { a (fixed) bounded operator. }
$$

Let us suppose, as we may, that in fact this isomorphism is the identity.

It is now a simple matter to introduce a right $A$-module structure and an $A$ valued inner product on $X(H)$ making it a right Hilbert $\mathrm{C}^{*}$-module over $A$ (using the result of Brown referred to). Let $s$ and $t$ be elements of $X(H)$, i.e., bounded uniformly continuous holomorphic sections of $H$, and let $a$ be an element of $A$.

Let us show that $s^{*} t$ belongs to $A$, in the natural sense (as a field of operators on the field of Hilbert spaces $\left(H(A)_{f}\right)_{f \in P_{0}(A)}$, on which $A$ acts) - so that we may then write $s^{*} t=(s \mid t)_{A}$ - and let us also show that $t a$ belongs to $X(H)$, i.e., that $t a$ is another bounded uniformly continuous holomorphic section of $H$. (Note that whether we consider $s^{*} t$ as acting on $H(A)_{f}$ for just the chosen $f$ in each unitary equivalence class in $P_{0}(A)$ or for all $f \in P_{0}(A)$ is immaterial, since the representations of $A$ corresponding to two unitarily equivalent elements of $P_{0}(A)$ are equivalent.) (Note also that, for each $f$, the description of the space of bounded holomorphic sections of $H$ over the equivalence class of $f$ as $B\left(H(A)_{f}, K\right)$ for a second Hilbert space $K$ is unique up to multiplication on the left by an isomorphism of Hilbert spaces - and so not changing the product $s^{*} t$ for two such sections $s$ and $t$ - since the subspace $B\left(H(A)_{f}, K\right) e$ must be (isometrically) preserved for each projection $e$ of rank one.)

To show that the bounded field of operators $s^{*} t$ belongs to $A$, by Theorem 6 of [3] we need only show that the function

$$
P_{0}(A) \ni f \mapsto f\left(s^{*} t\right) \in \mathbb{C}
$$

is uniformly continuous. (Here $f$ denotes also the canonical extension of $f$ to all bounded operators on the Hilbert space of $f$.) Since $s$ and $t$ and therefore also $s+i^{k} t$ for each $k \in\{0,1,2,3\}$ are uniformly continuous holomorphic sections of $H$, so that in particular the functions

$$
P_{0}(A) \ni f \mapsto f\left(\left(s+i^{k} t\right)^{*}\left(s+i^{k} t\right)\right)=\left\|s+i^{k} t\right\|_{f}^{2} \in \mathbb{R}
$$

$(k=0,1,2,3)$ are uniformly continuous, it follows by polarization, i.e., the identity,

$$
(\xi \mid \eta)=\frac{1}{4} \sum_{k=0}^{3} i^{k}\left\|i^{k} \xi+\eta\right\|
$$

(for any Hilbert space vectors $\xi$ and $\eta$ ), that $f \mapsto f\left(s^{*} t\right)$ is uniformly continuous, as desired.

To show that $t a$ is a bounded uniformly continuous holomorphic section of $H$ (given that $t$ is, and that $a$ belongs to $A$ ), since the section $t a$ is clearly bounded and holomorphic it is sufficient (by maximality - see Section 4) to show that the function

$$
P_{0}(A) \ni f \mapsto\|t a+s\|_{f} \in \mathbb{R}
$$


is uniformly continuous for any given bounded uniformly continuous holomorphic section $s$. Since

$$
\|t a+s\|_{f}^{2}=f\left(a^{*} t^{*} t a+a^{*} t^{*} s+s^{*} t a+s^{*} s\right),
$$

and since, as shown, $t^{*} t$ and $s^{*} t$ and therefore also $a^{*} t^{*} t a, s^{*} t a$, and $a^{*} t^{*} s(=$ $\left.\left(s^{*} t a\right)^{*}\right)$ belong to $A$, so that each of $f\left(a^{*} t^{*} t a\right), f\left(a^{*} t^{*} s\right), f\left(s^{*} t a\right)$, and $f\left(s^{*} s\right)$ depends uniformly continuously on $f$, it follows that also $\|t a+s\|_{f}$ depends uniformly continuously on $f$, as desired.

To check that the $A$-module structure $(t, a) \mapsto t a$ and the $A$-valued inner product $(s \mid t)_{A}=s^{*} t$ on $X(H)$ just constructed make $X(H)$ a Hilbert $A$-module, i.e., fulfil the conditions recalled at the beginning of the proof, recall from Section 4 that (by maximality) the space of bounded uniformly continuous holomorphic sections of a uniform holomorphic Hilbert bundle is complete in the supremum norm, and note that the norm $s \mapsto\left\|(s \mid s)_{A}\right\|^{1 / 2}=\left\|\left(s^{*} s\right)\right\|^{1 / 2}$ on the module is just exactly this supremum norm. (This amounts to noting that the two suprema $\sup _{f \in P_{0}(A)}\left(f\left(s^{*} s\right)\right)^{1 / 2}$ and $\sup _{f \in P_{0}(A)} f\left(\left(s^{*} s\right)^{1 / 2}\right)$ are equal —as $\left.\left\|s^{*} s\right\|^{1 / 2}=\left\|\left(s^{*} s\right)^{1 / 2}\right\|.\right)$

Note that the Hilbert $A$-module structure of $X(H)$ depends only on $H$-the $A$ valued inner product and $A$-module structure do not depend on the representation.

The next step is to show that, starting with the given Hilbert $A$-module $X$, one has a structure of uniform holomorphic Hilbert bundle on the set-theoretical bundle $H(X)=\left(H(X)_{f}\right)_{f \in P_{0}(A)}$ defined above, such that the canonical space of sections $X$ is included in the space of bounded uniformly continuous holomorphic sections. (It will follow from Section 4 that there is in fact a unique such structure-furthermore, as we shall see, the inclusion must be an equality!)

Recall from Section 4 that a structure of uniform holomorphic Hilbert bundle is based on a structure of holomorphic Hilbert bundle (uniquely determined by the uniform holomorphic structure). Let us describe what might be called the canonical holomorphic Hilbert bundle structure on $H(X)$. Let us note first that the Hilbert $A$ module structure of $X$ extends naturally (uniquely when weak* continuity is taken into account) to a Hilbert module structure on $X^{\prime \prime}$, the bidual of the Banach space $X$, over the bidual $A^{\prime \prime}$ of $A$ (also a $\mathrm{C}^{*}$-algebra, in fact a von Neumann algebra). The easiest way to see this is perhaps to view $X$ as the (lower) off-diagonal block of a $\mathrm{C}^{*}$-algebra - one diagonal block of which (the upper one) being $A$, and the other diagonal block (the lower one) being what might be called the left $\mathrm{C}^{*}$-algebra of $X$, namely, the norm-closed algebra of operators on $X$ generated by the operators $z y^{*}: x \mapsto z(y \mid x)_{A}, z$ and $y$ in $X$ (the *-operation mapping $z y^{*}$ into $y z^{*}$ - this Banach algebra with involution is well known to be a $\mathrm{C}^{*}$-algebra; see 12])- the two offdiagonal blocks being $X$ and what might be denoted by $X^{*}$, the space of elements of $X$ considered as maps from $X$ to $A$. (Surprisingly, this equivalent definition of a Hilbert $\mathrm{C}^{*}$-module, consisting of simply a realization of the given $\mathrm{C}^{*}$-algebra $A$ as a corner in a larger $\mathrm{C}^{*}$-algebra, determined by a multiplier projection, seems not to be current.) The Banach space bidual of this $\mathrm{C}^{*}$-algebra, when recognized again as a $\mathrm{C}^{*}$-algebra, gives rise immediately (as $A^{\prime \prime}$ is a multiplier corner of this bidual $\mathrm{C}^{*}$-algebra) to the desired Hilbert $A^{\prime \prime}$-module structure on $X^{\prime \prime}$.

In terms of the $A^{\prime \prime}$-module $X^{\prime \prime}$ the fibres $H(X)_{f}, f \in P_{0}(A)$, of $H(A)$ are easily recognized as the subspaces $X^{\prime \prime} e$ of $X^{\prime \prime}, e$ a minimal non-zero projection of $A^{\prime \prime}$. In the realization of $X^{\prime \prime}$ described above, one sees immediately that, for each $f \in P_{0}(A)$, the cutdown of $X^{\prime \prime}$ by the central support of $f$ in $A^{\prime \prime}$ is the space 
$B\left(H(A)_{f}, K_{f}\right)$ of bounded operators from the Hilbert space $\left.H(A)_{f}\right)$ to a second Hilbert space $K_{f}$. This yields a holomorphic Hilbert bundle structure of dual Hopf type, on the set-theoretical bundle $H(X)=\left(H(X)_{f}\right)_{f \in P_{0}(A)}$, as described in Section 3. With respect to this holomorphic Hilbert bundle structure, the holomorphic sections over the unitary equivalence class of a fixed non-zero $g \in P_{0}(A)$ are the maps

$$
e \mapsto t e
$$

from the space of projections of rank one on $H(A)_{g}$ to the space of operators $B\left(H(A)_{g}, K_{g}\right)$ with $K_{g}$ as above, with $t$ an element of the space $B\left(H(A)_{g}, K_{g}\right)$; a description of this holomorphic Hilbert bundle by means of local trivializations is given in Section 3.

Note that, with respect to the holomorphic Hilbert bundle structure on $H(X)$ just constructed, the sections in $X$ are holomorphic. Note also that these sections have uniformly continuous norm (with respect to the uniform structure on $P_{0}(A)$ arising from the pairing with $A$ ). By Zorn's lemma, $X$ is contained in a maximal such subspace - i.e., a subspace of the space of bounded holomorphic sections consisting of sections of uniformly continuous norm (the norm of the image in the fibre $H(X)_{f}$ depending in a uniformly continuous way on $f$ ) and maximal with this property. Choose such a subspace, and let us verify the remaining axiom of Section 4 for a uniform holomorphic Hilbert bundle, with respect to this subspace of sections. (As pointed out in Section 4, the purpose of this axiom is to ensure that the bounded uniformly continuous holomorphic sections determine the uniform holomorphic Hilbert bundle structure - including the holomorphic Hilbert bundle structure.)

Since already the smaller subspace of sections $X$ maps onto a dense subspace of the fibre $H(X)_{f}$ for each $f \in P_{0}(A)$ - by construction - and in fact onto the whole fibre by Kadison's transitivity theorem, as pointed out above (use the off-diagonal part of a $\mathrm{C}^{*}$-algebra description of a Hilbert $\mathrm{C}^{*}$-module given above), this is true for the chosen subspace, as desired.

In conclusion, the chosen subspace of bounded holomorphic sections (containing $X$, with each element of uniformly continuous norm, and maximal such) determines a structure of uniform holomorphic Hilbert bundle on $H(X)$ — of dual Hopf type as shown in the construction (of the underlying holomorphic Hilbert bundle).

We are now in a position (almost!) to show that the maximal subspace of bounded holomorphic sections with uniformly continuous norm chosen is equal to $X$. Namely, as shown earlier, this space is in a natural way a Hilbert $A$-module, which we may perhaps call $X(H(X))$. Note first that the Hilbert $A$-module $X$ is a sub Hilbert module of $X(H(X))$, in the sense that it is a submodule, and its $A$-valued inner product agrees with that constructed on $X(H(X))$ (as described above, beginning with the chosen holomorphic Hilbert bundle structure on $H(X))$. The submodule property follows from the compatibility of the inner products: granted the second property, and denoting by $x a$ the product of $x$ and $a$ in $X$, and by $x \cdot a$ the product of the same two elements, $x \in X$ and $a \in A$, in $X(H(X))$, we would have, for any $y \in X(H(X))$,

$$
(y \mid x a-x \cdot a)=(y \mid x a)-(y \mid x \cdot a)=(y \mid x) a-(y \mid x) a=0,
$$

where $x a=x \cdot a$ (choose $y=x a-x \cdot a$ ). Here, we have used the inner products for both Hilbert modules, and in particular have used the fact that $(y \mid x a)$ is the same computed in $X(H(X))$ (which is the only way the other two inner products 
$(y \mid x a-x \cdot a)$ and $(y \mid x \cdot a)$ make sense) and in $X$. To see that the inner product of two elements $x$ and $y$ of $X$ is the same in $X$ and in $X(H(X))$ recall that the bounded holomorphic sections of $H(X)$ are by definition the elements of the atomic type I part of the bidual $X^{\prime \prime}$, considered as a Hilbert $A^{\prime \prime}$-module, i.e., the direct summand $X^{\prime \prime} e_{I}$ of $X^{\prime \prime}$ where $e_{I}$ is the unit of the atomic type I part of the bidual $A^{\prime \prime}$ of $A$ (the sum of all minimal non-zero type I direct summands of this von Neumann algebra). Recall also that for each minimal non-zero central projection $p$ of $A^{\prime \prime}$ contained in $e_{I}$, the direct summand $X^{\prime \prime} p$ of $X^{\prime \prime}$ is the Banach space of all bounded linear operators between a certain pair of Hilbert spaces. Perhaps the best way to view the situation is to use the alternative definition of a Hilbert $A$-module given above, namely, the off-diagonal subspace $(1-e) B e$ of a $\mathrm{C}^{*}$-algebra $B$ with respect to a multiplier projection $e$ of $B$ such that the corner $e B e$ is equal to $A$ - the $\mathrm{C}^{*}$-algebra $B$ being uniquely determined if generated by $e B e$ and $(1-e) B e$. In particular let us use this description for the Hilbert $A$-module $X$ : namely, $X=(1-e) B e$ for a multiplier projection $e$ of a $C^{*}$-algebra $B$, such that the corner $e B e$ of $B$ is equal to $A$, and $B$ is generated as a $C^{*}$-algebra by $e B e$ and $(1-e) B e$. By definition of $B$, the inner product $(x \mid y)$ with respect to $X$ of two elements $x$ and $y$ of $X=(1-e) B e$ is $x^{*} y$. By construction, in terms of the present point of view, $X(H(X))$ is a subspace of the atomic type I part of $(1-e) B^{\prime \prime} e$, containing $X$, such that $x^{*} x$ belongs to $A \subseteq e B^{\prime \prime} e$ for every element $x$ of $X(H(X))$, and maximal with this property. The $A$-valued inner product of two elements $x$ and $y$ of $X(H(X))$ is, by definition, $x^{*} y$. In particular, one sees that the inner product in $X(H(X))$ agrees with the given inner product on the subspace $X$, as desired.

Next, note that the sub Hilbert module of $X$ of $X(H(X))$ has the property that, for every $f \in P_{0}(A)$, the corresponding direct summand of $X^{\prime \prime}$ (obtained by multiplying on the right by the central support of $f$ in $A^{\prime \prime}$ ) is equal to the a priori larger corresponding direct summand of $X(H(X))^{\prime \prime}$ (obtained by again multiplying on the right by the central support of $f$ in $A^{\prime \prime}$ ). This follows easily from the alternative definition of a Hilbert $A$-module given above, and just used, namely, the off-diagonal subspace $(1-e) B e$ of a $\mathrm{C}^{*}$-algebra $B$ with respect to a multiplier projection $e$ of $B$ such that the corner $e B e$ is equal to $A$. (And $B$ uniquely determined if generated by $e B e$ and $(1-e) B e$, as we shall assume it to be.) To see this, let us now view the Hilbert $A$-module $X(H(X))$ (rather than $X$ ) in this way, as the subspace $(1-e) B e$ of the $\mathrm{C}^{*}$-algebra $B$ (with $e B e=A$ ), and denote the sub- $C^{*}$-algebra of $B$ generated by the submodule $X$ of $X(H(X))$ by $B_{0}$. Note that the multiplier projection $e$ of $B$ is still a multiplier of $B_{0}$, and one has $(1-e) B_{0} e=X$ and $e B_{0} e=A$. Consider an irreducible representation of $A$ and its canonical extension to an irreducible representation of the larger $\mathrm{C}^{*}$-algebra $B_{0}$ (in which $A$ is contained as a multiplier corner, and in particular as a hereditary sub-C*-algebra). Think of the von Neumann algebra generated by $B_{0}$ as a minimal (non-zero) direct summand of type I of the Banach space bidual $B_{0}^{\prime \prime}$ of $B_{0}$. Recall that, by construction, not only $X$ but also $X(H(X))$ maps naturally into this direct summand of $(1-e) B_{0}^{\prime \prime} e\left(=\left((1-e) B_{0} e\right)^{\prime \prime}=X^{\prime \prime}\right)$-the elements of $X(H(X))$ are by definition sections of the field $H(X)$, the fibres of which are precisely the direct summands of $X^{\prime \prime}$ such as the one under consideration. (Recall also that $A$ maps naturally into $e B_{0}^{\prime \prime} e=A^{\prime \prime}$.) The key observation is that this map extends (uniquely) from $X(H(X))$ to a $\mathrm{C}^{*}$-algebra map from $B$ into the direct summand of $B_{0}^{\prime \prime}$ under consideration. (This holds because of what might be called the corner structure of 
$B$ with respect to the multiplier projection $e$ (namely, $B$ generated as a *algebra by $(1-e) B e=X(H(X))$ and $e B e=A)$.) This map then extends uniquely to a von Neumann algebra map from the bidual $B^{\prime \prime}$ onto the direct summand of $B_{0}^{\prime \prime}$ in question. In other words, the direct summands of $B_{0}^{\prime \prime}$ and of $B^{\prime \prime}$ corresponding to the representation of $A$ in question are equal. In particular, the direct summands in question of the Banach space duals $X(H(X))^{\prime \prime}\left(=(1-e) B^{\prime \prime} e\right)$ and $X\left(=(1-e) B_{0}^{\prime \prime} e\right)$ are equal, as asserted. (In fact, we shall only use the statement for $B^{\prime \prime}$ and $B_{0}^{\prime \prime}$.)

To show that $X$ is equal to $X(H(X))$, it is, of course, the same to show that $B_{0}$ is equal to $B\left(\right.$ as $X=(1-e) B_{0} e$ and $\left.X(H(X))=(1-e) B e\right)$. The second statement is more convenient as it fits more immediately into the theory of hereditary sub$\mathrm{C}^{*}$-algebras. Note that $B_{0}$ is a hereditary sub-C*-algebra of $B$, as there is a full hereditary sub- $C *$-algebra of $B_{0}$, namely, $A=e B_{0} e$, which is also hereditary in $B$. (In general, if $A_{1}$ is such a sub-C*-algebra of $A_{2}$, i.e., if there is a hereditary sub-C*algebra $A_{0}$ of $A_{1}$ which is full in $A_{1}$, i.e., not contained in a proper closed two-sided ideal of $A_{1}$, and which is hereditary also in $A_{2}$, then $A_{1}$ itself is hereditary in $A_{2}$. While we are not aware of a reference for this, the proof is short: Replacing $A_{2}$ by the hereditary sub-C*-algebra of $A_{2}$ generated by $A_{1}$, we may suppose that this is equal to $A_{2}$ and must then prove that $A_{1}$ is equal to $A_{2}$. It is sufficient to show that when the bidual of $A_{1}, A_{1}^{\prime \prime}$, is considered in the natural way as a subspace of $A_{2}^{\prime \prime}$, it is equal to it. Equivalently, we must show that in any representation of $A_{2}$, the images of $A_{1}$ and $A_{2}$ generate the same von Neumann algebra. This amounts to proving (note that in the case we are considering, $A_{1}^{\prime \prime}$ contains the unit of $A_{2}^{\prime \prime}$ ) that if $M_{1}$ is a sub von Neumann algebra of the von Neumann algebra $M_{2}$ (with the same unit), and if there exists a projection $p \in M_{1}$ with central support one in $M_{1}$ such that $p M_{2} p \subseteq M_{1}$, then $M_{1}=M_{2}$. This, of course, is the von Neumann algebra analogue of the $\mathrm{C}^{*}$-algebra lemma to be proved. While this, again, would not quite seem familiar, it is easily obtained by standard elementary von Neumann algebra methods. In the slightly special case that, in $M_{1}$, there exists an orthogonal family of equivalent projections with sum one, then it is a standard fact that $M_{1}$ is isomorphic to the tensor product of a von Neumann algebra isomorphic to $p M_{1} p$ and a factor of type $\mathrm{I}$, and, more precisely, is generated by $p M_{1} p$ and partial isometries between $p$ and the other projections in the orthogonal family. In particular, since at the same time $M_{2}$ is generated by $p M_{2} p$ and the same partial isometries, and $p M_{2} p=p M_{1} p$, this shows that -in this case- $M_{1}=M_{2}$. The general case is similar. (Choose by Zorn's Lemma a maximal orthogonal family of projections in $M_{1}$, equivalent to part of $p$, and one of them equal to $p$; then the sum of these projections is equal to one and $M_{1}$ is generated as a von Neumann algebra by $p M_{1} p$ together with partial isometries taking the other projections into subprojections of $p$. By the same token the larger von Neumann algebra $M_{2}$ is generated by $p M_{2} p$ together with the same partial isometries, and since $p M_{2} p=p M_{1} p$ it follows that $\left.M_{2}=M_{1}.\right)$ )

It remains to show, in order to prove that $X(H(X))=X$, that the hereditary sub-C*-algebra $B_{0}$ of $B_{1}$ is equal to $B_{1}$. It suffices to show that the open projection corresponding to $B_{0}$ in the bidual $B_{1}^{\prime \prime}$ of $B_{1}$, the unit of the bidual of $B_{0}$, is equal to the unit of $B_{1}$. We have shown that this is true inside each minimal non-zero direct summand of $B_{1}^{\prime \prime}$ of type I. Since the open projection in question belongs to the Borel algebra of $B_{1}^{\prime \prime}$, defined by Pedersen in [15] (as a subalgebra of $B_{1}^{\prime \prime}$ ), as does also the unit of $B_{1}^{\prime \prime}$, and since these two projections have the same image in 
each minimal non-zero type I direct summand of $B_{1}^{\prime \prime}$, by Corollary 3.9 of [15] these two projections are in fact equal, as desired.

In particular, the first specific statement of the theorem - as opposed to the general opening statement concerning equivalence of categories - is now established. Namely, we have shown that for each (right) Hilbert $A$-module $X$ the sections of the set-theoretic bundle of Hilbert spaces $\left(H(X)_{f}\right)_{f \in P_{0}(A)}$ defined in the statement of the theorem are (exactly) the bounded uniformly continuous holomorphic sections of a uniform holomorphic Hilbert bundle with these fibres, with respect to the canonical (Grassmannian) complex structure on $P_{0}(A)$ and the canonical uniform structure (corresponding to the pairing of $P_{0}(A)$ with $A$ ). According to Section 4, specifying the holomorphic uniformly continuous holomorphic sections of a uniform holomorphic Hilbert bundle with given fibres determines the holomorphic Hilbert bundle structure. As constructed, the bundle is of dual Hopf type, and by uniqueness it is therefore necessarily of dual Hopf type. Let us denote it by $H(X)$.

With regard to the second specific statement of the theorem, we have shown-in the course of the construction $X \mapsto H(X)$ - that for a given uniform holomorphic Hilbert bundle $H$, the space of bounded uniformly continuous holomorphic sections admits a canonical structure of right Hilbert $A$-module $X(H)$. (While, a priori, the $A$-valued inner product and $A$-module structure on the vector space $X(H)$ of bounded uniformly continuous holomorphic sections might be thought to depend on the identification of the space of all bounded holomorphic sections, restricted to a fixed equivalence class of $P_{0}(A)$, as $B\left(H(A)_{f}, K\right)$ for a representative element $f$ of this equivalence class and a second Hilbert space $K$-in which setting the sections are just the maps $e \mapsto t e$ for $\left.t \in B(H(A))_{f}, K\right)$ defined on the set of projections on $H(A)_{f}$ of rank one - it was pointed out above that the calculation of $s^{*} t$ in $B\left(H(A)_{f}\right)$ for two elements $s$ and $t$ in $B\left(H(A)_{f}, K\right)$ is independent of this identification, and, furthermore, the calculation of $s^{*} t$ in $B\left(H(A)_{f}\right)$ is independent of the choice of $f$, in the sense that for any two choices of a representative $f$ in the given equivalence class in $P_{0}(A)$ there is a unique isomorphism between the corresponding algebras of bounded operators $B\left(H(A)_{f}\right)$ preserving the canonical identification of the two calculations of $s^{*} t$. The unitary effecting this isomorphism between the two choices of $B\left(H(A)_{f}\right)$ is unique up to a scalar multiple, and so the isomorphism between the two choices of $B\left(H(A)_{f}, K\right)$ effected by this unitary is not quite unique, but the non-uniqueness can be realized by an automorphism of $K$, which as pointed out already leaves the calculation of $s^{*} t$ exactly unchanged.)

Not only is the map $H \mapsto X(H)$, like the map $X \mapsto H(X)$, canonical, but also, as is seen by inspection, $H(X(H))$ is equal to $H$, in the natural sense that the fibres of either bundle are just the natural quotients of $X(H)$, and the space of bounded uniformly continuous holomorphic sections is just, by definition, $X(H)$. (Note that according to the definition given in Section 4, a uniform holomorphic Hilbert bundle is determined by the space of its bounded uniformly continuous holomorphic sections.) It is immediate that (as asserted in the second statement of the theorem) the Hilbert $A$-module structure of $X(H)$ is determined uniquely by this property (i.e., $H(X(H))=H$ ): for any $x$ and $y$ in $X(H)$ the inner product $(x \mid y)$ is determined as an element of $A$ by the numbers $f((x \mid y)), f \in P_{0}(A)$, which constitute the Hilbert bundle structure of $H(X(H))$, and the module product ya is determined from this by the equation $(x \mid y a)=(x \mid y) a$. 
As we have seen, not only is $H(X(H))$ equal to $H$, for each uniform Hilbert module $H$ over $P_{0}(A)$ of dual Hopf type, but also, for each given Hilbert $A$-module $X, X(H(X))$ is equal to $X$.

It remains to check that the constructions $X \mapsto H(X)$ and $H \mapsto X(H)$ are functorial, with respect to the morphisms specified in the respective categories. This means, in the category of Hilbert $A$-modules, $A$-module maps which are bounded with respect to the canonical norm, and, in the category of uniform holomorphic Hilbert bundles over $P_{0}(A)$ (the pure state space of $A$ with 0 adjoined) - we are concerned only with the subcategory of bundles of dual Hopf type, but the morphisms are the same - uniformly bounded families (or fields) of bounded linear maps between corresponding fibres of uniform holomorphic Hilbert bundles $H_{1}$ and $H_{2}$, taking bounded uniformly continuous holomorphic sections of $H_{1}$ into bounded uniformly continuous holomorphic sections of $\mathrm{H}_{2}$.

Let $X_{1}$ and $X_{2}$ be Hilbert $A$-modules, and let $T$ be a morphism from $X_{1}$ to $X_{2}$. To obtain a morphism $H(T)$ from $H\left(X_{1}\right)$ to $H\left(X_{2}\right)$ - clearly in a functorial way - it is enough to show that, for each pure state $f$ of $A$, and each $x \in X_{1}$,

$$
f((T x \mid T x)) \leq\|T\|^{2} f((x \mid x)) .
$$

Indeed, it will follow immediately that $T$ induces a linear map $T_{f}$ from $H\left(X_{1}\right)_{f}$ to $H\left(X_{2}\right)_{f}$ of norm at most $\|T\|$. The field of maps $\left(T_{f}\right)_{f \in P_{0}(A)}$ will preserve bounded uniformly continuous holomorphic sections since these, as has been shown, are just the sections arising from the Hilbert module.

Note that the desired inequality, for fixed $x \in X_{1}$, and simultaneously for all $f \in P_{0}(A)$, is just the inequality

$$
(T x \mid T x)_{A} \leq\|T\|^{2}(x \mid x)_{A},
$$

where we have included the subscript $A$ to remind the reader that the inner products are elements of $A$.

On the other hand, it seems more direct just to prove the inequality separately for each $f \in P_{0}(A)$. Indeed, just as in the case of the Hilbert $A$-module $A$, the Hilbert space arising from an arbitrary Hilbert $A$-module corresponding to the pure state $f$ of $A$ is just the Banach space quotient of this module by the closed subspace associated to $f$ (the general case is seen from the special case on considering the $\mathrm{C}^{*}$ algebra description of the module) - and so the desired inequality exactly amounts to the property of $T$ that it preserves the closed subspace associated to each $f \in$ $P_{0}(A)$, i.e., takes the closed subspace $\left\{x_{1} \in X_{1} ; f\left(\left(x_{1} \mid x_{1}\right)\right)=0\right\}$ into the closed subspace $\left\{x_{2} \in X_{2} ; f\left(\left(x_{2} \mid x_{2}\right)\right)=0\right\}$. As we shall see, this property follows directly from the hypotheses on $T$ - that it is continuous and preserves the module structure. (In fact, as we shall see, continuity is not needed.)

It is sufficient to characterize the set

$$
X^{f}=\{x \in X ; f((x \mid x))=0\},
$$

for any given Hilbert $A$-module $X$ and any $f \in P_{0}(A)$, in terms of the module structure of $X$ (it would be acceptable to use the topology of $X$ too, but we shall not need to do so). Let us show that, simply,

$$
X^{f}=X A^{f},
$$

where $A^{f}$ is of course just $X^{f}$ in the case $X=A$. First, that $X A^{f}$ is contained in $X^{f}$ is immediate: $f\left(a^{*} a\right)=0$ implies $f((X a \mid X a))=f\left(a^{*}(X \mid X) a\right)=0$. 
Concerning the reverse inclusion, let us prove what is on the face of it stronger, that in fact

$$
X^{f}=X^{f} A^{f} .
$$

In the case $X=A$ this just follows from the statement $B^{2}=B$ for any $\mathrm{C}^{*}$-algebra, and the general case follows immediately from this case on considering (again) the $\mathrm{C}^{*}$-algebra description of the module.

Conversely, let $H_{1}$ and $H_{2}$ be uniform holomorphic Hilbert bundles over $P_{0}(A)$, of dual Hopf type, and let $T=\left(T_{f}\right)_{f \in P_{0}(A)}$ be a uniformly bounded field of linear mappings between corresponding fibres of $H_{1}$ and $H_{2}$ taking bounded uniformly continuous holomorphic sections of $H_{1}$ into uniformly continuous holomorphic sections (necessarily bounded) of $H_{2}$. We must show that $T$ is bounded considered in the natural way as a map from $X\left(H_{1}\right)$ to $X\left(H_{2}\right)$. It is sufficient to show the inequality considered above (but not forming part of the proof, yet):

$$
(T x \mid T x) \leq\|T\|^{2}(x \mid x), x \in X,
$$

where $\|T\|=\sup _{f \in P_{0}(A)}\left\|T_{f}\right\|$. As remarked above, for each $x \in X$ this is equivalent to proving

$$
f((T x \mid T x)) \leq\|T\|^{2} f((x \mid x)), f \in P_{0}(A),
$$

and for each $f \in P_{0}(A)$ this is just holds by assumption with $\left\|T_{f}\right\|$ in place of $\|T\|$.

This completes the proof of the theorem. It follows easily that the subcategories consisting of adjointable maps between Hilbert $A$-modules and morphisms between uniform holomorphic Hilbert bundles of dual Hopf type for which the Hilbert bundle adjoints are also such morphisms are also equivalent. Also one obtains an equivalence of categories on considering contractions (or isometries) alone.

Note that the fact that the functors $X \mapsto H(X)$ and $H \mapsto X(H)$ are inverses of each other (which holds it is clear at the level of maps as well as at the level of objects) implies immediately that each one is determined by the other. This may be compared with the more laborious but much sharper uniqueness statements in the theorem - namely, $H(X)$ is stated to be determined by the equation $X(H(X))=X$ before $X(H)$ is even defined for an arbitrary $H$, and in fact even for just a fixed given $X$, and then $X(H)$ is stated to be determined by the equation $H(X(H))=H$, but without referring to the equation $X(H(X))=X$ (which implies that $X \mapsto H(X)$ is injective), and in fact even for just a fixed given $H$ !

6.

The question arises how to characterize the pure state space of a $\mathrm{C}^{*}$-algebra. For instance, given a complex manifold such that each connected component is a Grassmannian - and at least one is just a point-, with a (complete) uniform structure which is coarser than that arising from the complex structure, can one simply say that it is enough for there to exist a uniform holomorphic Hilbert bundle of dual Hopf type - with no fibre equal to zero except at one of the Grassmannians consisting of a single point? (The distinguished point should then correspond to the zero functional in $P_{0}(A)$ for an eventual $\mathrm{C}^{*}$-algebra $A$ giving rise to the given complex and uniform structures.)

In any case, one has the following more elementary (if rather less elegant!) characterization: with a combined complex and uniform structure given as above, with the complex structure based on Grassmannians - and with at least one of these Grassmannians consisting of a single point, with one such point distinguished from 
the others - if one considers the direct sum of the Hilbert spaces corresponding to the Grassmannians - except with the zero Hilbert space at the distinguished pointand if one considers the self-adjoint norm-closed linear space of bounded families of linear operators on these Hilbert spaces which, when evaluated at the points of these Grassmannians (each such point giving a pure state of the $\mathrm{C}^{*}$-algebra of all bounded operators, except for the distinguished point which gives zero) yield uniformly continuous scalar-valued functions, and if one assumes that this linear space of families of operators is irreducible on each coordinate Hilbert space (not counting the zero one), and is closed under multiplication - so that it is a $\mathrm{C}^{*}$-algebra - say $A$ - , and if, moreover, the uniform structure on the given manifold coincides with that arising from the pairing with $A$-let us say that it is admissible if this is the case-, then this manifold is in a natural way identical to $P_{0}(A)$. (By the theorem of Brown in [3] used in the proof of Theorem 1, every $\mathrm{C}^{*}$-algebra arises in this way.)

The proof is quite simple - it is necessary only to show that every pure state of $A$ arises from a point in the manifold. Since the closed convex hull of the functionals arising from the manifold - either pure states or the zero functional - has supremum equal to the norm on every positive element of $A$, it follows that this is the convex set of all positive functionals of norm at most one on $A$. By Appendice B.14 of [4, the set of all pure states of $A$ must be contained in the closure of the set of pure states under consideration, together with zero. Since by assumption, the uniform structure on the given manifold coincides with that arising from the pairing with $A$, and furthermore is complete, it follows that all the pure states of $A$ arise from points of the manifold, as asserted.

\section{REFERENCES}

1. S. Dineen, Complex Analysis on Infinite Dimensional Spaces, Springer-Verlag, London, 1999. MR 1705327 (2001a:46043)

2. V. Brînzănescu, Holomorphic Vector Bundles over Compact Complex Surfaces, SpringerVerlag, Berlin, 1996. MR:1439504 (98g:32053)

3. L. G. Brown, Complements to various Stone-Weierstrass theorems for $\mathrm{C}^{*}$-algebras and a theorem of Shultz, Comm. Math. Phys. 143 (1992), 405-413. MR1145802 (93c:46113)

4. J. Dixmier, Les $\mathrm{C}^{*}$-algèbres et leurs représentations, Gauthier-Villars, Paris, 1964. MR0171173 (30:1404)

5. J. M. G. Fell, The structure of algebras of operator fields, Acta Math. 106 (1961), 233-280. MR.0164248 (29:1547)

6. R. Godement, Théorie générale des sommes continues d'espaces de Banach, C. R. Acad. Sci. Paris 229 (1949), 1321-1323. MR0029102 (10:548b)

7. R. Godement, Sur la théorie des représentations unitaires, Ann. of Math. 53 (1951), 68-124. MR0038571 (12:421d)

8. K. K. Jensen and K. K. Thomsen, Elements of KK-Theory, Mathematics: Theory \& Applications, Birkhäuser, Basel, 1991. MR1124848 (94b:19008)

9. I. Kaplansky, Modules over operator algebras, Amer. J. Math. 75 (1953), 839-858. MR 0058137 (15:327f)

10. G. G. Kasparov, Hilbert $\mathrm{C}^{*}$-modules: theorems of Stinespring and Voiculescu, J. Operator Theory 4 (1980), 133-150. MR.587371 (82b:46074)

11. K. Kawamura, Serre-Swan theorem for non-commutative $\mathrm{C}^{*}$-algebras, J. Geom. Phys. 48 (2003), 275-296. MR2007596 (2004h:46086)

12. E. C. Lance, Hilbert $C^{*}$-modules. A toolkit for operator algebraists, London Mathematical Society Lecture Note Series 210, Cambridge University Press, Cambridge, 1995. MR.1325694 (96k:46100)

13. V. M. Manuilov and E. V. Troitsky, Hilbert C*-modules. Translations of Mathematical Monographs, 226. American Mathematical Society, Providence, R. I., 2005. MR 2125398 (2005m:46099) 
14. W. L. Paschke, Inner product modules over B*-algebras, Trans. Amer. Math. Soc. 182 (1973), 443-468. MR0355613 (50:8087)

15. G. K. Pedersen, Applications of weak* semicontinuity in $\mathrm{C}^{*}$-algebra theory, Duke Math. J. 39 (1972), 431-450. MR0315463(47:4012)

16. M. A. Rieffel, Induced representations of $\mathrm{C}^{*}$-algebras, Advances in Math. 13 (1974), 176-257. MR0353003 (50:5489)

17. F. W. Shultz, Pure states as a dual object for C*-algebras, Comm. Math. Phys. 82 (1982), 497-509. MR641911 (83b:46080)

18. J. Tomiyama and M. Takesaki, Applications of fibre bundles to the certain class of $\mathrm{C}^{*}$-algebras, Tôhoku Math. J. (2) 13 (1961), 498-522. MR0139025(25:2465)

Department of Mathematics, University of Toronto, Toronto, Canada M5S 2E4

E-mail address: elliott@math.toronto.edu

Department of Mathematics, Ritsumeikan University, Kusatsu, Shiga 525-8577, Japan

E-mail address: kawamura@kurims.kyoto-u.ac.jp 